\section{PEDIATRIC EDITORIAL}

\section{Pediatric Highlights}

International Journal of Obesity (2006) 30, 1.

doi:10.1038/sj.ijo.0803170

This issue marks the second anniversary of Pediatric Highlights in the IJO. Since this feature started, over 60 papers on pediatric obesity have been highlighted in the quarterly issues in which the Pediatric Highlights appear. Overall, the number of papers on pediatric obesity submitted to IJO has grown dramatically since the pediatric focus issues were instituted. The Editors are very pleased that the Pediatric Obesity community has adopted IJO as their specialty journal of choice and we look forward to a continued close relationship.

This issue of IJO continues the tradition of excellence in Pediatric Obesity with a number of papers focusing on the relationship of childhood obesity and complications that heretofore have been associated with adult obesity, body composition in childhood, and treatment for childhood obesity. Asthma, atherosclerosis, and metabolic syndrome are increased in obese children, just as they are in obese adults. The topic of body composition measurement methods in pediatric patients is developing rapidly. Significant physical changes occur during the years spanning infancy through young adulthood that make accurate body composition measurements problematic. In this issue, the relationship of waist circumference to measures of total and visceral adipose tissue is described in a review by Brambilla et al. Finally, Steinbeck et al. have presented a proposed protocol for a prospective meta-analysis on the treatment of childhood obesity, which we hope will be of interest to the many researchers who are interested in management of childhood obesity. These papers point out the limitations of current knowledge in pediatric obesity, the need to reduce the lifelong burden of obesity-related disease, and point the way to additional research in this critical specialty.

RL Atkinson ${ }^{1}$ and I Macdonald ${ }^{2}$ Editors

${ }^{1}$ Obetech Obesity Research Centre, Virginia Biotechnology Research Park, 800 East Leigh Street, Richmond, VA 23219, USA

and

${ }^{2}$ Institute of Clinical Research, School of Biomedical Sciences, University of Nottingham, Clifton Boulevard, Nottingham, NG7 2UH, UK

E-mails: ratkinson2@mail2.vcu.edu; ian.macdonald@nottingham.ac.uk 